corroborating evidence of UGIB are very unlikely to have active bleeding and/or the need for endoscopic haemostasis. These patients could have an UGI capsule endoscopy in the emergency department as opposed to hospital admission and subsequent wait for an inpatient OGD. This could consequently have significant implications on admission rates, LOS and hospital associated morbidity.

\section{P362 DEVELOPMENT OF THE FIRST UK LICENCED FAECAL MICROBIOTA TRANSPLANT SERVICE: RESULTS OF FIRST YEAR'S ACTIVITY}

${ }^{1} S$ Shabir*, ${ }^{2} \mathrm{D}$ Inglis*, 'S Manzoor, ${ }^{1,2} \mathrm{MN}$ Quraishi, ${ }^{1} \mathrm{C}$ Green, ${ }^{1,2} \mathrm{~N}$ Sharma, ${ }^{1,2} \mathrm{~T}$ Iqbal. ${ }^{1}$ University of Birmingham Microbiome Treatment Centre, Birmingham, UK; ${ }^{2}$ Department of Gastroenterology, Queen Elizabeth Hospital, University Hospitals Birmingham NHS Foundation Trust, Birmingham, UK

\subsection{6/gutjnl-2020-bsgcampus.436}

Introduction The use of Faecal Microbiota Transplant (FMT) has become established practice for the treatment of recurrent or refractory Clostridiodes difficile infection (CDI). It involves the transplant of minimally processed donor faecal material into a recipient's GI tract. Regulatory change in 2015 saw FMT classed as a medicinal product in The Human Medicines Regulations. As recognised by joint British Society of Gastroenterology and Healthcare Infection Society guidelines, these regulatory requirements are more readily fulfilled by a specialist centre, utilising a supply and satellite model - akin to that adopted by the Microbiome Treatment Centre (MTC). The MTC, supplies FMT on a named-patient basis under a Manufacturer's Specials' (MS) licence. FMT is supplied under the 2019-2020 NHS England Innovation and technology payment tariff, zero-cost model.

Methods The MTC strictly screens donors. Dedicated laboratory facilities are used for FMT production, minimising the risk of cross-contamination and allowing standardisation of the production process. FMT is stored frozen, allowing for multiple samples to be obtained immediately after donor screening. A numbering system is used in conjunction with a treatment directory to track FMT preparations. A multidisciplinary team approach is adopted, with screening, production and deployment overseen by a clinical gastroenterologist, microbiologist, service and production managers. Each FMT request is assessed for its clinical indication and then discussed with the requesting clinician.

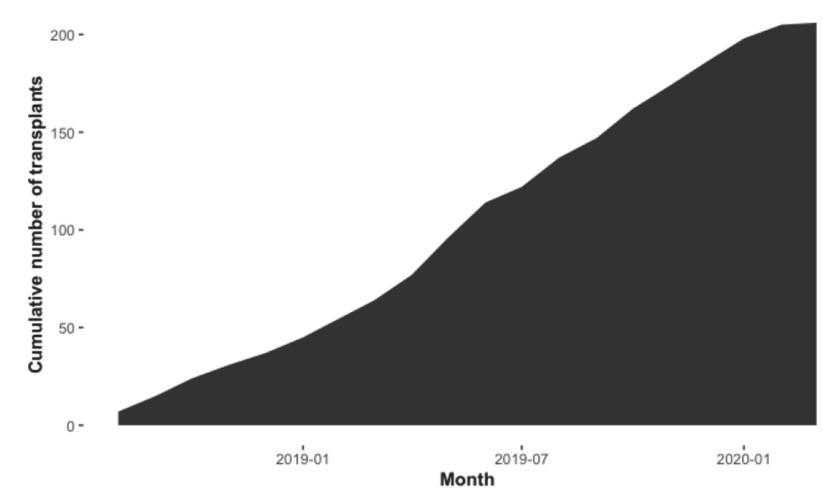

Abstract P362 Figure 1 Cumulative FMTs (by month)
Results From August 2018-January 2020, 181 FMT treatments have been given to 159 patients in 61 hospitals around the UK for recurrent or refractory CDI (Abstract P362 figure 1). 139 of these patients received a single FMT treatment with 20 patients receiving further transplants. Clinical follow up data has been received for $79 \%(n=110)$ of patients receiving a single FMT treatment. There has been an $81 \%$ $(n=89)$ rate of reported resolution of CDI at 7 days posttransplant.

Of the 20 patients requiring more than 1 FMT, 4 had showed resolution of symptoms at 7 days post both first and second transplant, suggesting re-infection. All requested FMTs have been supplied to the clinical sites for the requested treatment date $(25 \%$ were supplied in under 24 hours, 34\% within $24-48$ hours and $40 \%$ in over 48 hours.)

Conclusions The further development of the UK's first FMT service has greatly improved NHS access to this novel technology, with 45 NHS trusts using the service in the past year, compared to 21 the year previously. FMT material is supplied expediently, with the majority arriving with the requesting clinician in under 48 hours.

\section{P363 STARTING AZATHIOPRINE IN IBD: PRESCRIBE A THERAPEUTIC DOSE OR START LOW AND INCREASE SLOWLY?}

Diluka Karunaratne* ${ }^{*}$ Andrew Phillips, Francesca Melindo, Neil Hawkes, James Berrill. Royal Glamorgan Hospital, Llantrisant, UK

\subsection{6/gutjnl-2020-bsgcampus.437}

Introduction Azathioprine is frequently prescribed for the management of inflammatory bowel disease (IBD), however there is considerable variation in how it is initiated. It remains common practice to start patients on low dose Azathioprine and gradually increase the dose, with the belief that this approach will improve drug tolerance. However, this process can take several weeks or months to reach a therapeutic drug level, requiring multiple blood tests and patient contacts.

Since April 2018, patients attending our IBD clinic who require Azathioprine, have been started directly on to a therapeutic $2 \mathrm{mg} / \mathrm{kg}$ dose. Outcomes from this 'therapeutic dose group' of patients (TDG), in terms of drug tolerability and safety, are compared to those from patients that started Azathioprine prior to April 2018, who were started on a low dose and increased gradually (LDG).

Methods In this single-centre, retrospective, observational study, adult IBD patients starting on Azathioprine between March 2016 and November 2019 were identified using pharmacy records. Patients with low thiopurine methyltransferase (TPMT) level, and patients who had taken thiopurine medication previously were excluded from the study.

The proportion of patients successfully established on Azathioprine was compared between the two groups, with a 'success' defined as continuing a therapeutic dose of medication beyond three months. Reasons for stopping Azathioprine prior to this were recorded.

Results The proportion of patients successfully established on Azathioprine was similar in both groups, with $54 \%$ in the LDG $(n=63)$ compared to $66 \%$ in the TDG $(n=29)$. There 